\title{
A Perspective Approach (OABC) Algorithm using Square Odd Routing for minimized Energy Consumption
}

\author{
Akrati Sharma $^{1}$, V. P. Sharma ${ }^{2}$, Hitesh Sharma ${ }^{3}$ \\ ${ }^{I}$ Computer Science \& Engineering, Govt. Engineering College, Ajmer, India \\ ${ }^{2}$ Computer Science \& Engineering, Assistant Professor Govt. Engineering College, Ajmer, India \\ ${ }^{3}$ Computer Science \& Engineering, Govt. Engineering College, Ajmer, India \\ 1akratininna@gmail.com, ${ }^{2} v$ p.ecajmer@gmail.com, ${ }^{3}$ hiteshsharma0123@gmail.com.
}

\begin{abstract}
ABC set of principles has been already proposed furthermore with some drove guidelines, yet the length of the work parameter has been spinning round detecting the hubs in static or dynamic way with no accentuation at the power consumption parameter ideal from the crossing hubs vitality to warmth dissemination control or the entire charge for navigating or detecting every one of the hubs or the time admission for the hub records to get transmitted from one hub to each other. Our proposed work is essentially constructed generally in light of the sending of the system however now not arbitrarily but rather to some extent construct absolutely with respect to parameters. Our work proposed a totally one of a kind and favored an approach of $\mathrm{ABC}$ calculation for unpracticed detecting of actualities with regards to control looking after age. The proposed set of guidelines guarantees the identification of implied objective however with the ideal strategy with diminished vitality utilization.

The ABC has a few applications for choosing the optical neighboring arrangement construct absolutely for the most part with respect to swarm knowledge. Our paper concentrates on the versatile system of $\mathrm{ABC}$ calculation with the versatile approach of the square and strange statute so as to pick the most appropriate course of unpracticed transmission. Our papers have changed the conventional approach of the honey bee state to keep successively in the meantime as not having any unique measurements. We have proposed an unpracticed switch based absolutely convention in WSN. We have proposed the square odd technique to improve the conventional strategy in the more prominent dynamic way. The considered arrangement of principles should offer rest to trade hubs in the meantime the unmistakable hub is vigorous. Our proposed procedure will definitely decrease the quality utilization and at the indistinguishable time will bring about looking through the related most astounding decent bearing to embellish the test results fabulously.
\end{abstract}

Index Terms-ABC, WSN, bee colony,square odd

\section{INTRODUCTION}

$\mathrm{W}$ I-FI Bee Colony Optimization is a met heuristic in light of the ordinary searching practices of bumble bees. Exactly when a bumble bee adequately finds sustenance, it returns to the hive and grants the position and partition to the support source to hive mates by methods for a waggle move, which was not grasped until decoded by Karl von Frisch in 1974. The waggle move is a figure of eight move, and grants bearing as the move's point to the sun and the length of the central region of the move is particularly comparing to the division to the sustenance. Distinctive hive mates by then have a choice, they can take after another bumble bees' turn and go to a comparable support source, where they may find more sustenance sources, or to examine aimlessly (which in nature is astoundingly phenomenal). Once the craftsman has completed its turn it can either watch another bumble bees move, or return to the sustenance source it advanced.

Bumble bee System was first exhibited in [LT01], which incited the change of Bee Colony Optimization and its application to the Traveling Salesman Problem (TSP) in [WL$\mathrm{C} 08 \mathrm{a}]$. The met heuristic is a swarm knowledge approach, which implies it is depicted by individuals doing excess exercises and a clear specific method between individuals, realizing iterative difference in course of action quality. Bumble bee Colony Optimization has been used to strike a grouping of issues, including TSP [WLC08b, WLC09a, and WLC09b] and the p-center issue [TDS11]. The bumble bee area headway met heuristic has four phases consistent with age, and the estimation accentuates till some circumstance is met. The game plan of methodologies has an outrageous and fast of cutting edge bumble bees, the wide range dependent upon the issue and trouble case, paying little mind to the path that among 20 and 50 is standard. The data is the main look at into memory, normally as some style of matrices and the game plan of standards starts off developed.

Each age joins the going with four phases:

Game plan creating. Each bumble bee builds up an answer the utilization of the course of action picked in arrange four of the past age (beside it is the essential new release, this is communicated later) and some burden correct resources (close by side period in tsp).

Daemon exercises. Bother particular advancements and neighborhood looks are continuing running in this phase to enhance the courses of action. The blend of optimistic and gathering are chasing down methods licenses making more grounded results. 
Feature it 'waggle move'. Bumble bees put it accessible authentic surprising responses for each other to be used as a piece of later accentuations. Look at 'waggle move'. Each bumble bee assembles courses of action build totally upon an in the past created plan. In this movement, every get this sort of an answer, both by methods for the usage of the game plan is created inside the past age or with the guide of following a great game plan made with the guide of method for some other bumble bee station.

\section{BACKGROUND}

Honey bee settlement streamlining is a met heuristic in view of the normal rummaging practices of honey bees. In the meantime as a honey bee adequately uncovers nourishment, it comes back to the hive and imparts the position and separation to the suppers source to hive-buddies through a waggle move, which altered into never again comprehended until decoded by method for the use of Karl von Frisch in 1974. The waggle move is an observer of 8 moves and imparts course in light of the fact that the move's point of view to the sun oriented and the span of the focal period of the move is on the double relative to space to the sustenance. Other hive mates at that point have a want, they could pick to accommodate with some other honey bees' move and travel to the equivalent dinners source, in which they'll find more prominent suppers resources, or to find arbitrarily (which in nature is extremely phenomenal). As fast in light of the fact that the artist has finished its move it can each watch some unique honey bees move, or backpedal to the supper's source it promoted.

Honey bee gadget changed into first included [LT01], which prompted the improvement of honey bee state enhancement and its application to the visiting businessperson inconvenience (tsp) in [WLC08a]. The met heuristic is a swarm insight approach, which implies it is portrayed by utilizing the utilization of individuals doing monotonous developments and a simple correspondence method between individuals, bringing about an iterative change of arrangement best.

\section{OVERVIEW OF OBJECT TRACKING}

$\mathrm{ABC}$ set of rules has been beforehand proposed moreover with a couple of drove ideas, yet the length of the papers parameter has been rotating around detecting the hubs in static or dynamic path with none accentuation on the power admission parameter ideal from the navigating hubs quality to warmth scattering power or the entire rate for crossing or detecting the greater part of the hubs or the time utilization for the hub data to get transmitted from one hub to each extraordinary. Our proposed paper depends on the arrangement of the system however now not haphazardly but rather halfway construct absolutely for the most part with respect to parameters. The one's parameters are as per the following - :

The calculation needs to give an upgraded come about to directing in a dynamic way and delight in the hubs in a unique way. The arrangement of rules must be dependable for extensive kind of hubs detected through and through with less vitality utilization. The proposed set of rules should bounty significantly less time to eat as dispose of the non-reacting system.

Case 1: The proposed work has never again indiscriminately thought about every one of the hubs coming in the gathering in evaluation to the customary procedure of (ABC) set of standards the proposed calculation has contemplated wonderful the exchange hubs coming inside the method for steering correspondences.

Case2: The proposed papers has created power productive outcomes the measurement which winds up not considered inside the present procedure .we've consolidated the idea of tradition $(\mathrm{ABC})$ set of controls by means of checking sensors in the square and strange mode to survey the vitality unpracticed surrender the final product.

Case 3: The considered approach has proposed another idea for $(A B C)$ set of standards with a direct substitute for the customary approach for assessing and supplants quality admission. the proposed set of guidelines alluded to as upgraded $\mathrm{ABC}(\mathrm{OABC})$ set of principles, that is remarkable in its strategy from blessing system in every one of the parameters we've expressed previously.

The organizations and float along with execution and grade by review depiction of the proposed set of tenets are one of a kind as takes after. The proposed calculation is adjusted with steering and power green streamlining convention utilizing manufactured honey bee settlement $(\mathrm{ABC})$ calculation,

Stage 1: The system has been instated first to get the hubs enacted a decent approach to position into impact (ABC) set of rules

Stage 2: The group can be sent first on the off chance that you need to feel the reasonable way to have the capacity to save vitality.

Stage 3: The accompanying advance is to check sensors in the rectangular and weird mode to assess the vitality unpracticed stop result.

Stage 4: Look at counterfeit honey bee state $\mathrm{ABC}$ - so on bunches to find the streamlining course among hubs to last areas.

Stage 5: The pondered strategy has proposed a current thought for $(A B C)$ set of directions with the direct exchange in customary method for assessing and replaces control utilization

Stage6: Investigate whether all hubs wind up detected if certain at that point indicate group ways of life time and move bring down back else hold to stage three. 


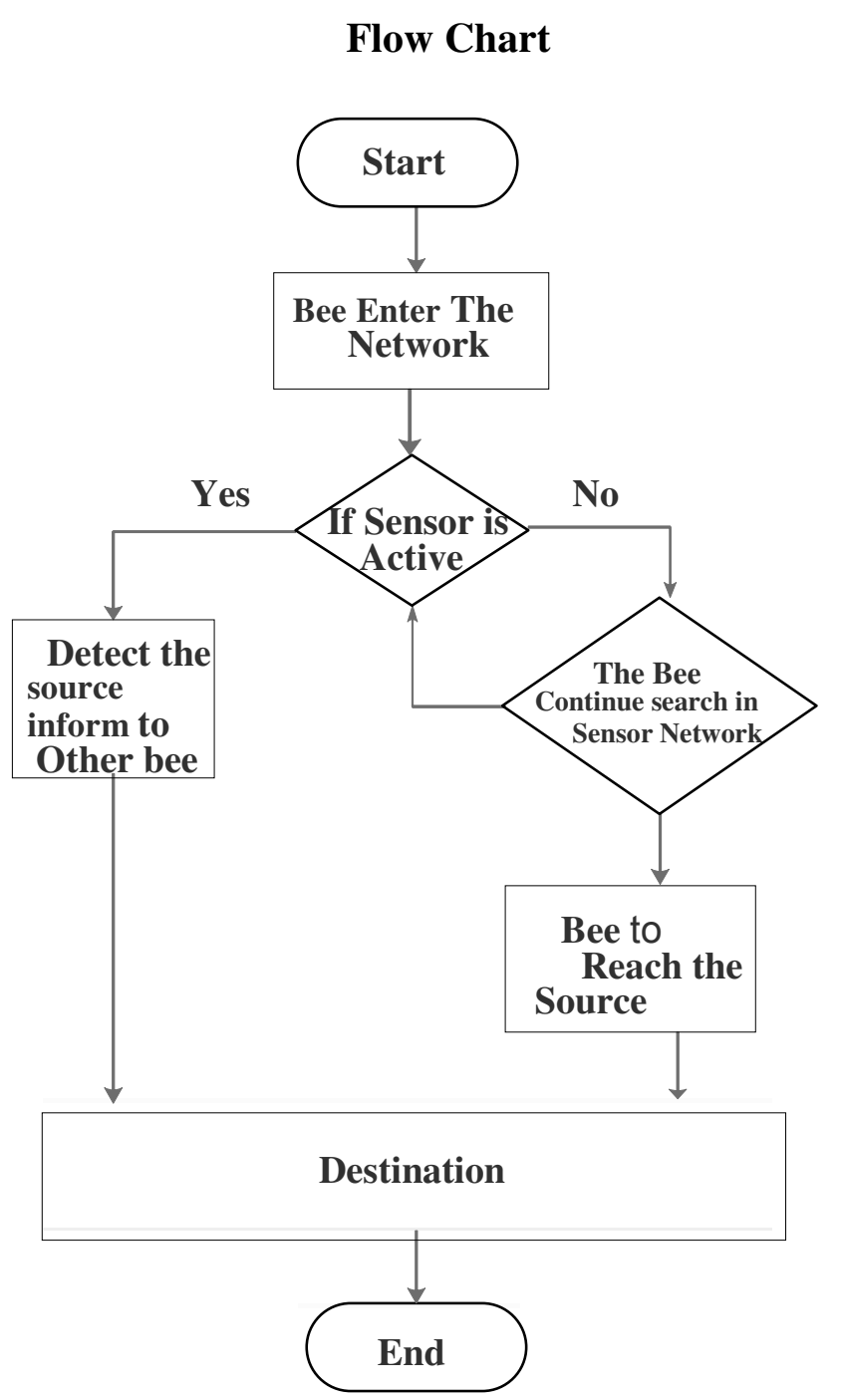

IV. SIMULATION AND RESULTS

Simulation is a to a great degree essential present day advancement. It's far going to be refined to absolutely correct age, outlining, or opportunity programming program fields for various capacities. pc motor helped recreation will variation hypothetic and certifiable nearness contraptions or diversions practices wears on a versatile workstation basically so it will be concentrated to check however the device works. The ns-2 simulation condition offers exceptional flexibility in examines the attributes of sensor sorts out in view of it starting at now fuses versatile plans for quality confined Wi-Fi amazing frameworks. Inside the ns-2 condition, a sensor framework might be worked with a couple of unclear contraptions of traditions and affinities as those available in the around the world.
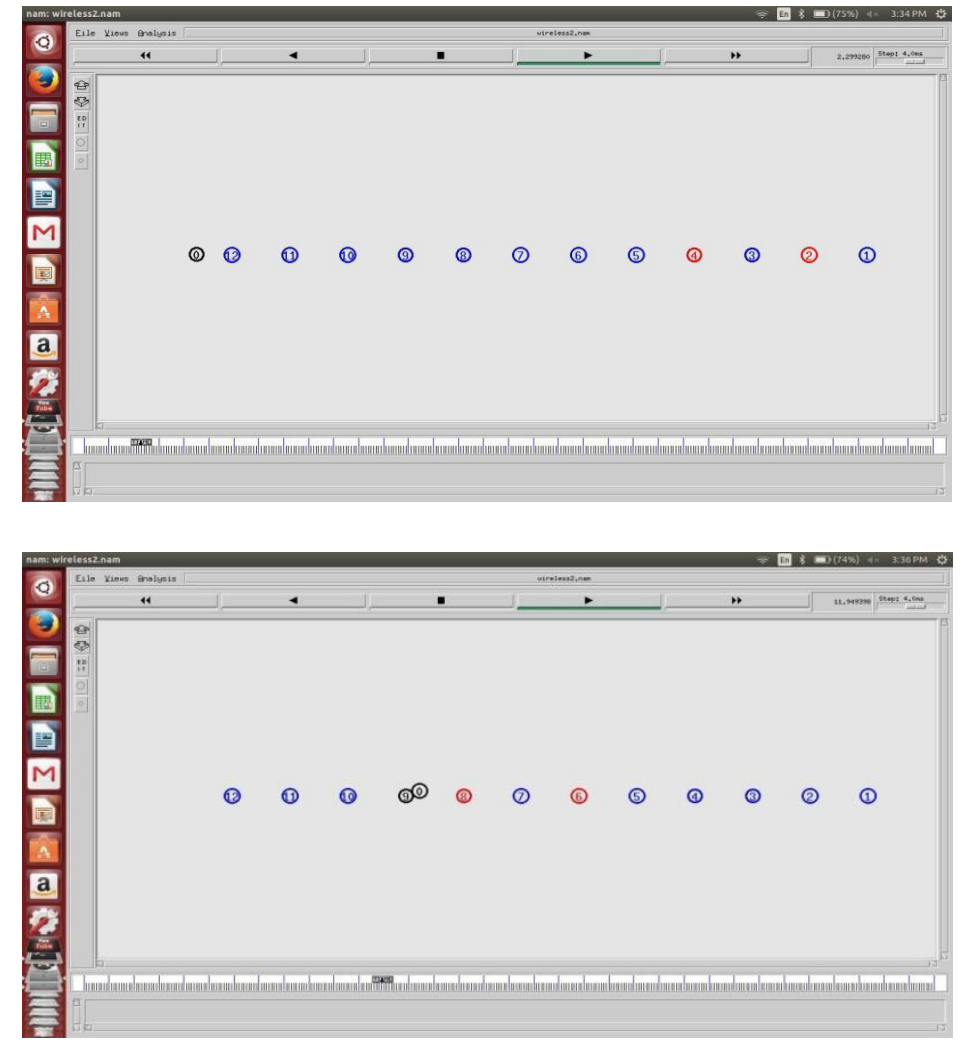

\section{CONCLUSION AND FUTURE WORK}

Our paper proposed a specific and fine approach of $\mathrm{ABC}$ set of tenets for green identifying of records concerning imperativeness securing age. The proposed estimation guarantees the revelation of expected target however with most time-tested strategy with diminished essentialness use. The paper is done up with the execution consequences of proposed Algorithm for $\mathrm{ABC}$ for square unusual recognizing the particular center points dynamically. The dynamic strategy for $\mathrm{ABC}$ set of fundamentals impacts it to appropriate for the specific guiding tradition to get related with for destiny applications. the proposed set of controls has now not a singular estimation filling in as it is proper with other controlling tradition counts and it's miles uncommonly a lousy bundle appropriate in the specific zone like surveillance machine, street framework, and interference IDs.

\section{REFERENCES}

[1] Sheikhpour, Razieh, and Sam Jabbehdari. "A two-level cluster based routing protocol for wireless sensor networks." International Journal of Advanced Science and Technology, Vol. 45, pp.19-30, August 2012.

[2] Manikandan, A., and S. Rathinagowri. "Wireless Sensor Networks: A Research Perspective Survey." International Journal of Technology Enhancements and Emerging Engineering Research, VOL 1, ISSUE 3, pp. 9-11, 2013. 
[3] Kaur Lovedeep, and Rajneesh Talwar. "Review for Leach Protocol in WSN" International Journal of Advances in Science and Technology, Vol. 2, Issue 4, pp. 31-33, 2014.

[4] Deepali Virmani, Tanu Sharma and Ritu Sharma, " Adaptive Energy Aware Data Aggregation Tree for Wireless Sensor Networks ". International Journal of Hybrid Information Technology Vol. 6, No. 1, pp. 26-36, January, 2013.

[5] Ji, Sai, Liping Huang, and Jin Wang. "A Distributed and Energyefficient Clustering Method for Hierarchical Wireless Sensor Networks." International Journal of Future Generation Communication and Networking, Vol. 6, Issue 2, pp. 83-92, 2013.

[6] Zhang, Haitao, Shiwei Zhang, and Wenshao Bu. "A Clustering Routing Protocol for Energy Balance of Wireless Sensor Network based on Simulated Annealing and Genetic Algorithm." International Journal of Hybrid Information Technology, Vol.7, Issue 2, pp. 71-82, 2014.

[7] Ma, Teng, Yun Liu, and Zhen-jiang Zhang. "An Energy-Efficient Reliable Trust-Based Data Aggregation Protocol for Wireless Sensor Networks." International Journal of Control and Automation Vol. 8, Issue 3, pp. 305-318, 2015.

[8] S. Kumar, T. H. Lai, and J. Balogh., "On K-Coverage in a Mostly Sleeping Sensor Network,” in MOBICOM. ACM, 2011.

[9] D. Tian and N. Georganas, "A Node Scheduling Scheme for Energy Conservation in Large Wireless Sensor Networks," Wireless Communications and Mobile Computing Journal, May 2011.

[10] J. Jeong, Y. Gu, T. He, and D. Du, "VISA: Virtual Scanning Algorithm for Dynamic Protection of Road Networks," in Proc. of 28th IEEE Conference on Computer Communications (INFOCOM 09), Rio de Janeiro, Brazil, April 2009.

[11] M. Mar 'oti, B. Kusy, G. Simon, and 'Akos L'edeczi, “The Flooding Time Synchronization Protocol," in SENSYS. Baltimore, Maryland, USA: ACM, Nov. 2004
[12] L. Lazos, R. Poovendran, and J. A. Ritcey, Analytic evaluation of target detection in eterogeneous wireless sensor networks," ACM Trans. Sensor Networks, vol. 5, no. 2, pp. 1-38, March 2009.

[13] J. Hwang, T. He, and Y. Kim, "Exploring In-Situ Sensing Irregularity in Wireless Sensor Networks," in SENSYS. ACM, Nov. 2007, pp. 289-303.

[14] Y. Gu and T. He, "Data Forwarding in Extremely Low Duty-Cycle Sensor Networks with Unreliable Communication Links," in SENSYS. Sydney, Australia: ACM, Nov. 2007, pp. 321-334.

[15] J. Hwang, T. He, and Y. Kim, "Exploring In-Situ Sensing Irregularity in Wireless Sensor Networks," in SENSYS. ACM, Nov. 2007, pp. 289-303.

[16] Y. Gu and T. He, "Data Forwarding in Extremely Low Duty-Cycle Sensor Networks with Unreliable Communication Links," in SENSYS. Sydney, Australia: ACM, Nov. 2007, pp. 321-334.

[17] G. Lu, N. Sadagopan, B. Krishnamachari, and A. Goel, "Delay Efficient Sleep Scheduling in Wireless Sensor Networks," in INFOCOM. IEEE, 2005.

[18] D. Marpe, H. Schwarz, T. Wiegand, S. Bosse, B. Bross, P. Helle, T. Hinz, H. Kirchhoffer, H. Lakshman, N. Tung; S. Oudin, M. Siekmann, K. Suhring, M. Winken, "Improved video compression technology and the emerging high efficiency video coding standard", 2011 IEEE International Conference on Consumer Electronics (ICCE 2011), pp. 52-56, 2011.

[19] M. H. Sedky, M. Moniri, C. C. Chibelushi, "Classification of smart video surveillance systems for commercial applications", IEEE Conference on Advanced Video and Signal Based Surveillance (AVSS 2005), pp. 638-643, 2005.

[20] T. V. Dung, Q. N. Truong, "Quality Enhancement for Motion JPEG Using Temporal Redundancies", IEEE Transactions on Circuits and Systems for Video Technology, Volume: 18 , Issue: 5, pp. 609-619, 2008 